\title{
Influence of lateral embayments on suspended sediment transport under unsteady flow conditions
}

\author{
Carmelo Juez ${ }^{1, *}$, Matthias Thalmann ${ }^{1}$, Anton J. Schleiss ${ }^{1}$ and Mário J. Franca ${ }^{1,2}$ \\ ${ }^{1}$ École Polytechnique Fédérale de Lausanne (EPFL), Laboratoire de Constructions Hydraulique (LCH), \\ Lausanne, Switzerland \\ ${ }^{2}$ IHE-Delft Institute for Water Education, Delft, The Netherlands
}

\begin{abstract}
Local widening in a channelized river is a common practice in restoration projects. The lateral embayments built for this purpose in the river banks are partially filled up by fine sediments. This allows the formation of aquatic habitats with hydraulic and morphologic diversity. However, the design of these lateral cavities may be compromised by the fluctuations in the water discharge. To address this problem, systematic experimental investigations have been carried out with five different fluctuating hydrograph scenarios. Water depth, sediment concentration and area covered by the settled sediments are analysed in each experiment. The process of sedimentation in the lateral embayments proved to be, in general, resilient to flow fluctuations. However, there were several differences observed during the high flow phase fluctuation phase depending on the geometric configuration of the embayments and the applied hydrograph: (i) a higher peak in discharge means that more sediments are resuspended and a complete remobilization of the in-cavity sediment deposits is possible. (ii) Long and short cavities are more resilient to high flow events than cavities with an intermediate length.
\end{abstract}

\section{Introduction}

Within the framework of river restoration projects, local widening in a river reach is commonly considered for providing shelters for the plants and animals of the riparian habitat. This restoration approach causes the appearance of areas with low velocity where the fine sediments transported in suspension face a sudden drop in the turbulence intensity. The fine sediments are thus trapped in the in-cavity regions. This sedimentation process has a vital importance due to the relevant role that fine sediments play in the biodiversity of the riparian habitats: attached to them, there are nutrients (mainly phosphates and nitrates) that help in the algae growth.

Consequently, the study of the local widening in a river reach (also called lateral embayments or cavities) and its impact on the main flow has received serious attention along

* Corresponding author: carmelo.juez@epfl.ch 
the years [1-5]. Nevertheless, most of these earlier works were focused on: the study of the hydrodynamic patterns and the feedback generated between the main flow and the lateral cavities. Only little attention was drawn to the analysis of the role of sediment transport under these conditions.

In a previous study, [6], the influence of the lateral cavities on the transport of suspended fine sediments in the main channel was studied considering a constant flowrate. Depending on the geometrical design of the lateral embayments configurations, cavities were filled up partially or. Based on this previous work, the aim of this study is to go a step further. That is, whether the in-cavity sediment deposits will be flushed during the flow fluctuation phase or whether they will remain. This question is of paramount importance since all the rivers are influenced by flow fluctuations that control the sediment distribution patterns. These fluctuations may have: (i) a natural origin, e.g. the winter-summer flow patterns or (ii) an anthropogenic origin, as the so-called hydropeaking. In both cases, river organisms and their habitat are impacted. Without appropriate flow shelter habitat, the fluctuating regime can affect over-wintering survival rates. Furthermore, if the majority of the fine material trapped inside the lateral embayment is remobilised during an episode of high discharge a turbidity increase may occur which can difficult fish visibility and thus impairing visual feeding behaviour.

To tackle the impact of flow regime fluctuations, several studies have been carried out in the past, each one targeting at least one key element of the river life: sediment patterns, [7], hydropower operation models , [8], hydraulic magnitudes (wet areas, water levels, shearstress), [9] and riparian vegetation or fish, [10]. However, and despite of these previous efforts, the impact of lateral embayments behaving as buffer areas filled with sediments and bearing potential side effects is still unknown. For this reason, in this work, systematic experimental investigations in a straight channel have been carried out with different lateral embayment configurations that were proven to be suitable for restoration projects. Starting at baseflow until a quasi-equilibrium condition was reached, five different scenarios with an increased discharge were applied. After a specific period, the initial baseflow was again imposed in the channel. Thanks to this setup the following ideas have been addressed in this work: (i) which type of lateral embayment completely or partially shelters the sediments already trapped, (ii) which is the impact of the different hydrograph scenarios in terms of time duration, peak discharge, and ramping rate on this morphological process, and (iii) check if the sedimentation and subsequent remobilisation of the sediments is a reversible process, i.e. whether after the increase in the discharge it is possible to reach the same sedimentation patterns as before the high flow phase.

\section{Experimental setup}

\subsection{Flume description}

Experiments were performed in a hydraulic sys-tem that works in a closed circuit with different components, including: (i) an upstream tank (length $\mathrm{x}$ width $\mathrm{x}$ height $=2 \mathrm{mx} 1 \mathrm{~m} \mathrm{x}$ $1 \mathrm{~m}$ ) that serves as a mixing tank for the suspended sediments, (ii) a rectangular open channel ( $7.5 \mathrm{~m} \mathrm{x} 1 \mathrm{~m} \times 1 \mathrm{~m}$ ) with $0.1 \%$ slope (typical slope for the subcritical flows in Alpine valleys), and (iii) a downstream tank $(3.5 \mathrm{~m} \times 1 \mathrm{~m} \times 1 \mathrm{~m})$ that collects the circulating flow. The water is then externally pumped from the downstream to the upstream tank. The channel bottom is smooth and made of painted wood. Figure 1 shows a sketch of the experimental setup. 


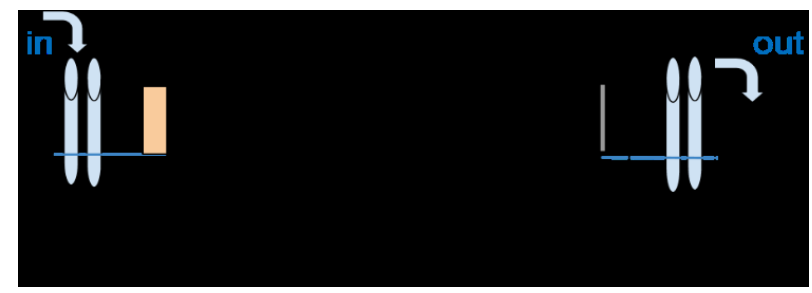

Fig. 1. Sketch of the experimental flume.

\subsection{Lateral embayment configurations}

The straight base channel was modified by adding cement bricks $(0.25 \mathrm{~m} \times 0.10 \mathrm{~m} \times 0.19 \mathrm{~m})$ at the side of the channel against the lateral walls. This allowed to build different lateral embayment configurations as it was already done in $[6,11]$. These large-scale depressions in the lateral banks of the channel are characterised by the length of the cavity (1), the longitudinal distance between two cavities (L) and the lateral depth of the cavities (W). The combination of these characteristic lengths of the lateral embayments together with the channel width leads to the definition of several geometrical ratios: the expansion ratio $(\mathrm{ER}=$ $(b+2 \mathrm{~W}) / \mathrm{b})$, the aspect ratio $(A R=W / 1)$, and the roughness ratio $(\mathrm{RR}=\mathrm{W} / \mathrm{L})$.

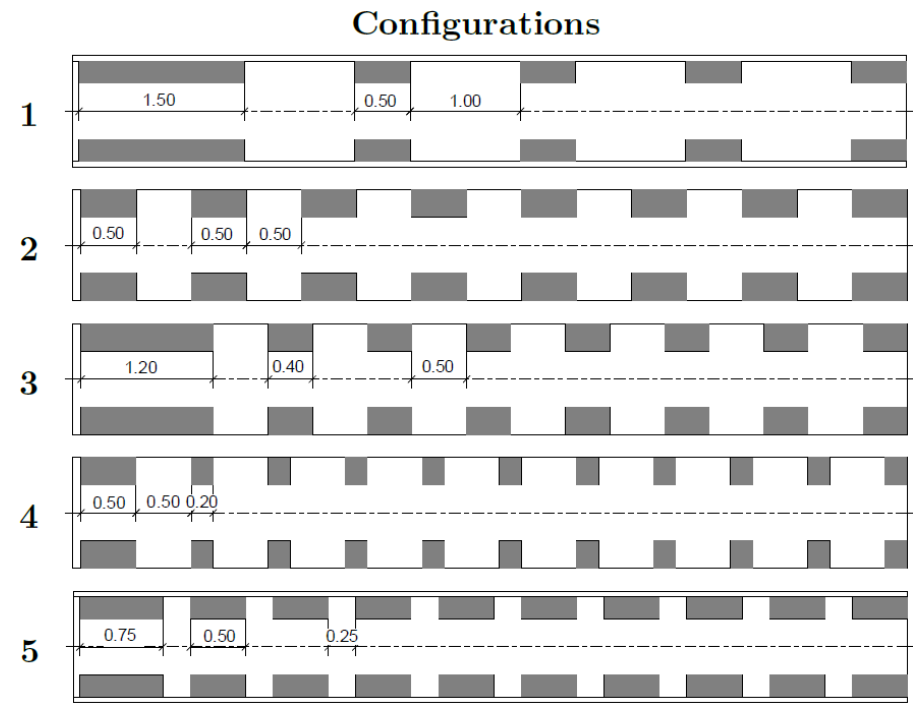

Fig. 2. Geometric configurations tested with detailed definition of the geometric lengths in meters.

The lateral embayment configurations tested in this work were based on a prior experimental campaign carried out by [6]. Five lateral embayment configurations were chosen and are displayed in Figure 2. Their geometric parameters are shown in Table 1. From configuration 1 to 5, they have increasing AR, i.e. a decreasing length of the cavities. The three configurations with the same $\operatorname{AR}(2,3$ and 4$)$ have increasing RR, i.e. a decreasing space between the cavities. As it was concluded in [6], configurations with high ER and AR are advantageous for restoration purposes. They were partially filled-up with sediments, thereby promoting the appearance of areas with low and high velocity, suitable for aquatic biota. 
Table 1. Summary of the test ranges of the geometrical parameters.

\begin{tabular}{cccc}
\hline Configuration & ER $[-]$ & AR $[-]$ & RR $[-]$ \\
\hline 1 & 1.67 & 0.20 & 0.40 \\
2 & 2.00 & 0.50 & 0.50 \\
3 & 2.00 & 0.50 & 0.60 \\
4 & 2.00 & 0.50 & 1.20 \\
5 & 1.67 & 0.80 & 0.40 \\
\hline
\end{tabular}

\subsection{Suspended sediment}

The material conveyed in suspension within the flow was Polyurethane artificial sediments. The properties of this material are: a grain size of $\mathrm{d}_{50}=0.2 \mathrm{~mm}$, a density $\rho_{\mathrm{s}}=1160 \mathrm{~kg} / \mathrm{m}^{3}$ and a uniformity of 0.47 . The mean diameter of particles was chosen to be in the range of non-cohesive fine sediment, $0.062-0.500 \mathrm{~mm}$. An initial concentration was chosen to fulfil the maximum suspended capacity of the flow, i.e. $\mathrm{c}_{0}=0.5 \mathrm{~g} / \mathrm{l}$.

\subsection{Suspended sediment}

Five different hydrograph scenarios have been tested in each lateral embayment configuration. They are shown in Figure 3. These scenarios include differences in: (i) the duration of the increased discharge: one (L1, H1 and S1) or two hours (L2 and H2); (ii) the maximum discharge applied: $8.5 \mathrm{l} / \mathrm{s}$ (L1 and L2) or $15.0 \mathrm{l} / \mathrm{s}(\mathrm{H} 1, \mathrm{H} 2$ and S1), and (iii)the ramping rate of flow variations: immediate increase (L1, L2, H1 and H2) or an increase of $0.34 \mathrm{l} / \mathrm{s}$ per minute (S1). After the high flow phase, the initial base flow was imposed for two hours in order to study the morphological answer of the system during the recovery time.

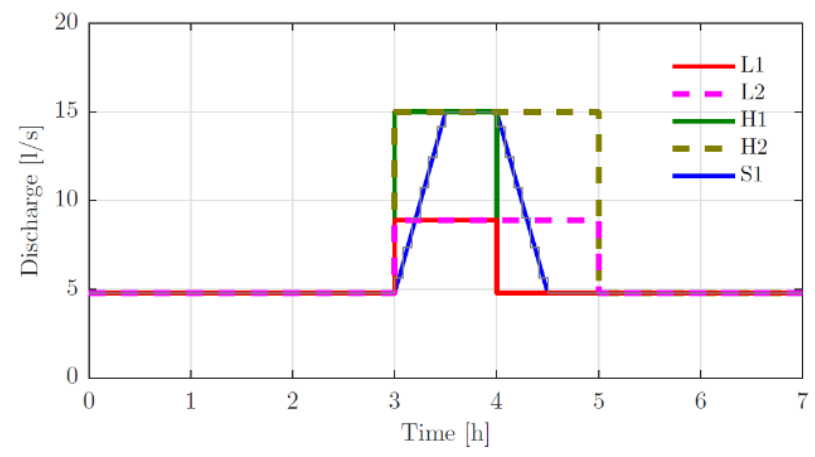

Fig. 3. Applied hydrograph scenarios.

\subsection{Flow conditions}

Table 2 displays the hydrodynamic variables measured and calculated for each scenario. Maximum discharges applied in each scenario were chosen keeping in mind the relation between the water height ( $h$ ), and the channel width (b), being the shallowness ratio $S R=b / h$ between 7.2 and 17.3. Such way, shallow flow conditions were guaranteed. Therefore, it is adequate to assume that the turbulence structures that appear in each experiment have a quasi$2 \mathrm{D}$ nature and are in consequence the net contributors for the mass exchange between the main flow and the lateral embayments [1]. The flow was turbulent and subcritical in all the experiments. The water depth along the channel was constant since the channel was run under uniform flow conditions. 
Table 2. Summary of flow conditions for each discharge tested.

\begin{tabular}{lccc}
\hline Discharge $[1 / \mathrm{s}]$ & $\mathrm{Q}_{1}=4.8$ & $\mathrm{Q}_{2}=8.5$ & $\mathrm{Q}_{3}=15.0$ \\
\hline Water depth $[\mathrm{m}]$ & 0.035 & 0.050 & 0.070 \\
Velocity $[\mathrm{m} / \mathrm{s}]$ & 0.25 & 0.32 & 0.39 \\
Froude number $[-]$ & 0.43 & 0.46 & 0.47 \\
Reynolds number [-] & 30968 & 54754 & 86907 \\
\hline
\end{tabular}

\section{Experimental techniques}

\subsection{Suspended sediment monitoring}

The temporal evolution of the suspended sediment concentration was recorded by turbidimeters in two locations of the channel: upstream and downstream. However, an adaptation length was set $(3 \mathrm{~m}$ upstream after the flow tranquiliser and $1 \mathrm{~m}$ downstream before the gate) in order to avoid any perturbation in the measurements so that the sediment concentration profile was well dispersed within the flow. Information provided by the turbidimeters is punctual and it corresponds to a value in the vertical concentration profile. This height has been chosen in order to ensure that the value measured is roughly the averaged value of the vertical sediment concentration profile. This height also prevents the presence of air bubbles under the turbidimeters and avoids possible signal reflection from the bottom that may induce measurement errors.

\subsection{Sediment deposition pattern}

The study of the influence of the sedimentation on the cavity was performed all through the experiments following a twofold protocol: (i) plan view photos of the sedimentation patterns were taken in a cavity placed in between the two turbidimeters. Subsequently, these photos were treated for extracting the surface occupied by the depositions by using the characteristic sediment pixel value for filtering the image. (ii) The second variable measured was the total sediment mass trapped inside the lateral embayments. Later, these sediment samples were dried in an oven to determine the dry weight. This mass was divided by the total area occupied by the embayments in order to compute the trapping efficiency in mass per area for each configuration at the end of the experiments. For the sake of brevity these results are not showed in this work.

\section{Results}

\subsection{Suspended sediment concentration}

Figure 4 shows the sediment concentration in the main channel normalised by its initial value $(0.5 \mathrm{~g} / \mathrm{l})$ for three selected embayment configurations with three selected hydrograph scenarios. Each experiment starts with three hours of low discharge. During this time, the channel reaches a quasi-equilibrium state where changes in suspended sediment concentrations and in sediment deposits are only marginal. The high flow phase is then imposed in the channel. This leads to an increase in sediment concentration due to the remobilisation of the in-cavity sediment deposits. After the high flow phase, the initial flowrate is imposed in the channel and a new morphological equilibrium is obtained.

As it is displayed in Figure 4, larger differences in the sediment concentration decay for the different phases of the hydrograph scenarios are observed. The concentration decay is 
slower during the high flow phase than during the base flow phase. This tendency is more evident for larger differences in the flow rate (H1 and S1) than for lower differences (L2). The concentration decay rates before and after the high flow phase are almost the same for all experiments.
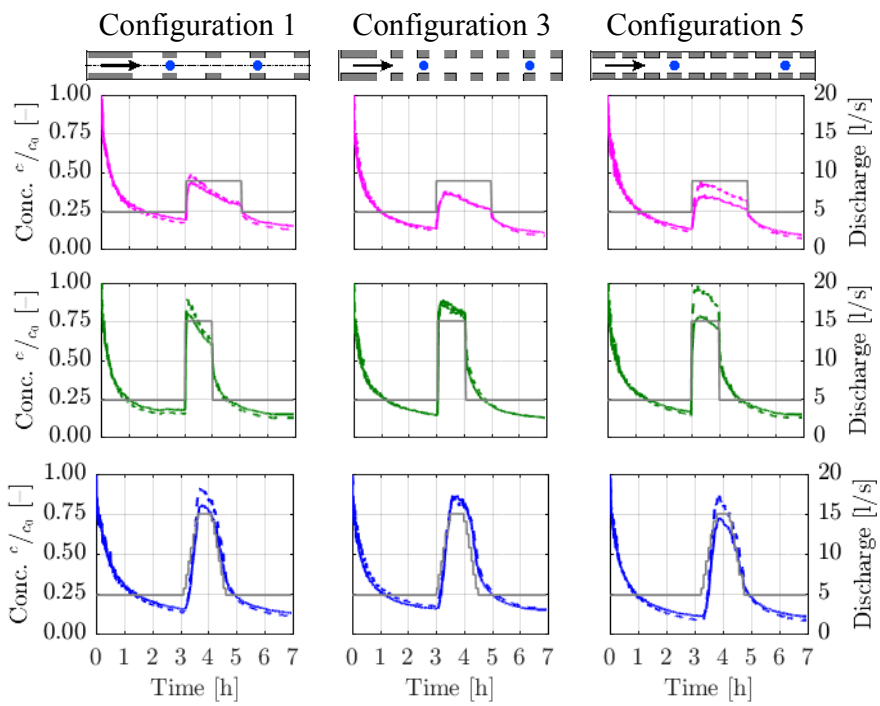

Fig. 4. Normalized sediment concentration for all experiments. The colored solid line represents the concentration at turbidimeter 1 (upstream), the dashed line the concentration at turbidimeter 2 (downstream) and the thin grey line the discharge. The experiments for configurations 1, 3 and 5 (left to right) are displayed according to the small figures in the head line with flow direction according to the arrows. The blue dots represent the positions of the turbidimeters in flow direction. The high flow phase events L2, H1 and S1 are shown (from top to bottom).

\subsection{Sediment deposition area}

Figure 5 illustrates the temporal evolution of the area covered by the sediments, normalized by the area of the cavity. For configuration 1, the decrease in the sediment deposition area is low for all the hydrograph scenarios. The sediment patterns in this configuration are resistant to changes in the discharge, even to peak flows of high magnitude (L2, H1 and S1). Configuration 5 shows a high resistance of the deposits to be flushed away for hydrograph scenarios L2 and S1. Configuration 3 displays a low resistance to the high flow phase. For small changes in discharge (L2), the deposit areas decrease. However, for the larger flow changes (H1 and S1), the cavities are completely flushed away.

After the high flow phase events, the sediment deposits in the cavities are recovered. Therefore, the resilience of the system to the high flow phase is high. This means that the process is reversible and that after the high flow phase, the in-cavity deposition deposits obtained before the high flow phase are restored.

On the other hand, Figure 6 shows the temporal evolution of the sediment deposition area for four specific instants in one representative cavity. These four specific instants are explained in the caption of the figure. Visual observation confirms that sediment deposits in configuration 1 are more resilient to be re-mobilized due to the high flow phase. 


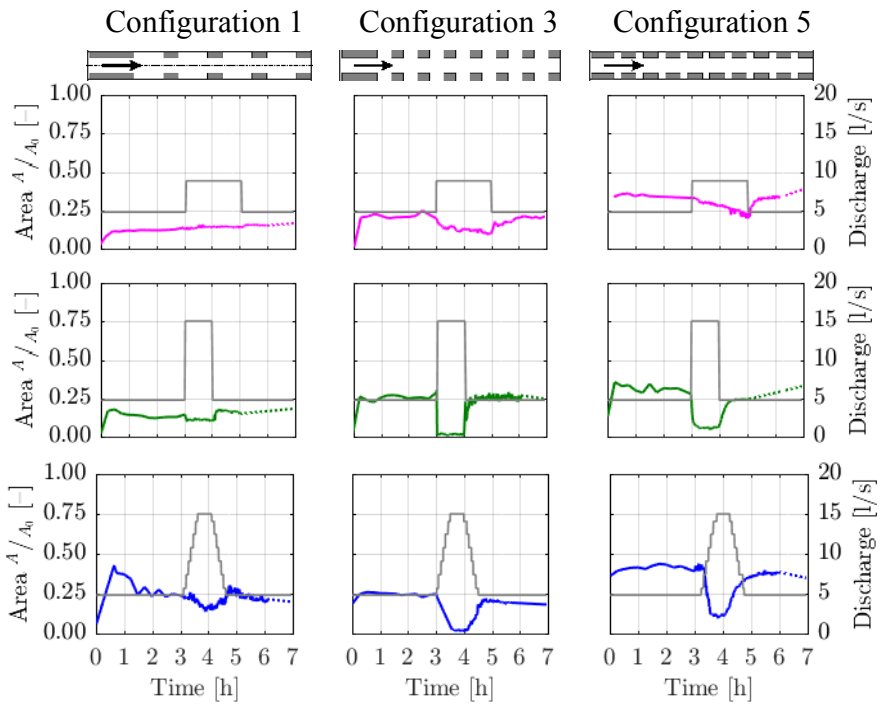

Fig. 5. Temporal evolution of the sediment deposition area for different configurations. The colored line represents the area of the sediment deposit in the observed cavity and the thin grey line the discharge. The dotted lines link the last value of the period with continuous photography with the area of the final deposition. The experiments for configurations 1, 3 and 5 (left to right) are displayed according to the small figures in the head line with flow direction according to the arrows. The high flow phase events L2, H1 and S1 are shown (from top to bottom).
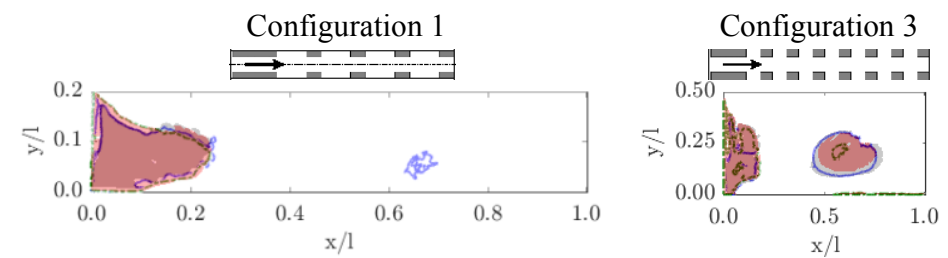

Configuration 5
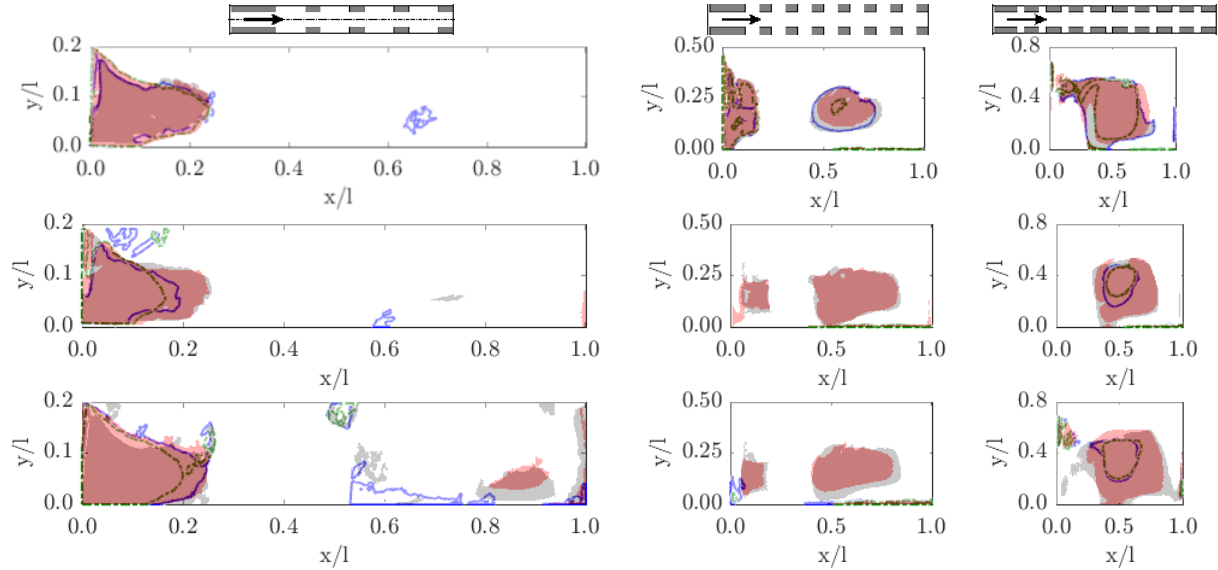

Fig. 6. Temporal evolution of the in-cavity sediment deposition area for four specific instants in one cavity. The grey shaded areas represent the area occupied by sediments $15 \mathrm{~min}$ before the high flow phase event. The blue and green contour lines indicate the sediment deposition area 5 min before and 5 min after the high flow phase, respectively event. The red shaded areas show the extension of the sediment deposits $60 \mathrm{~min}$ after the end of the high flow phase. Configurations 1, 3 and 5 (left to right) are displayed according to the small figures in the head line with flow direction according to the arrows. The high flow phase events L2, H1 and S1 are shown (from top to bottom). 


\section{Conclusions}

The results of this study show that the in-cavity sediment deposits in a channel equipped with lateral embayments are highly resilient to flow fluctuations. The dynamics of the in-cavity sediment deposits are influenced by two factors: (i) the cavity aspect ratio (AR), it determines the impact of the high flow phase. For long cavities (low AR), the dead water zone is large and persistent enough to keep a large fraction of the deposits settled, even at high peak discharges. The depositions in very short cavities (high AR) are quite resistant to high flow phase too as they are widely decoupled from the flow in the main channel and can maintain a high suspended sediment concentration for a long time which feeds the deposits despite the high turbulent forces. For intermediate AR, the depositions remain largely unsheltered from the high flow phase. (ii) The magnitude of the hydrograph plays a vital role as well. The peak discharge determines the impact of the high flow phase since the amount of material resuspended is directly linked to it. The duration of the high flow phase event and the ramping rate are less relevant.

This work was funded by the FOEN (Federal Office for the Environment, Switzerland) in the framework of the research project Sediment and Habitat Dynamics.

\section{References}

1. W.S.J. Uijttewaal, D.V Lehmann, A.V. Mazijk. J. of Hydr. Eng., 127(11), 928-936 (2001).

2. V. Weitbrecht, S.A. Socolofsky, G.H. Jirka. J. of Hydr. Eng., 134(2):173-83 (2007).

3. M.F.M. Yossef, H.J. de Vriend. J. Hydr. Eng. 136(2), 610-625 (2010).

4. Y. Akutina. Experimental investigation of flow structures in a shallow embayment using 3D-PTV. PhD Thesis. McGill University, Montréal (2015).

5. E. Mignot, W. Cai, G. Launay, N. Rivière, C. Escauriaza. Phys. of Fluids, 28:045104. (2016).

6. C. Juez, I. Buehlmann, G. Maechler, A.J. Schleiss, M.J. Franca. Earth Surf. Process. Landf. 43(1):271-284 (2017).

7. N. Lana-Renault, D. Regués. Earth Surf. Process. Landf, 34:1291-1301 (2009).

8. T. Meile, J.-L. Boillat, A.J. Schleiss. Aquatic Sciences, 73:171-182 (2011).

9. S. Spiller, N. Rüther, H. Friedrich. Water, 7:4951-4970, (2015).

10. J.M. Ribi, J.-L. Boillat, A. Peter, A.J. Schleiss. Aquatic Sciences, 76(4):527-541 (2014).

11. T. Meile, J.-L. Boillat, A.J. Schleiss. J. of Hydr. Eng., 137(12):1588-1597 (2011). 